tains the connected set $W-W \times C^{\prime}$. But since we also know that $M=W^{\prime}=\left(W-W \times C^{\prime}\right)^{\prime}+C^{\prime},\left(W-W \times C^{\prime}\right)^{\prime}=M$ and so $Z_{1}$ contains $Z_{2}$, which is impossible. Therefore $W-C$ is connected and so $W$ is the sum of two mutually exclusive connected subsets, which is a contradiction. Hence $W$ must be widely connected.

Ohio State University

\title{
QUADRILATERALS INSCRIBED AND CIRCUMSCRIBED TO A PLANE CUBIC*
}

BY A. H. DIAMOND

In a paper by $\mathrm{M}$. W. Haskell $\uparrow$ the geometrical configurations of triangles inscribing and circumscribing a plane cubic curve have been studied by analytic methods. The purpose of this paper is to examine the properties of quadrilaterals inscribing and circumscribing a plane cubic curve by means of elliptic functions.

The coordinates of a point on the curve will be expressed in terms of Weierstrass' elliptic functions $\wp(u)$ and $\wp^{\prime}(u)$. It is known that $3 n$ points of the cubic are the points of intersection of the cubic with a curve of order $n$ if $\ddagger$

$$
u_{1}+u_{2}+\cdots+u_{3 n} \equiv 0 \bmod \left(\omega_{1}, \omega_{2}\right) .
$$

The values of the parameters of the vertices of the quadrilaterals are obtained from a consideration of the congruences

$$
2 u_{1}+u_{2} \equiv 0,2 u_{2}+u_{3} \equiv 0,2 u_{3}+u_{4} \equiv 0,2 u_{4}+u_{1} \equiv 0,
$$

whence

$$
15 u_{1} \equiv 0,
$$

or

$$
u_{1}=\frac{k_{1} \omega_{1}+k_{2} \omega_{2}}{15}
$$

* Presented to the Society, November 29, 1930.

$\dagger$ Haskell, this Bulletin, vol. 25 (1918), p. 194.

$\ddagger$ Appell and Lacour, Théorie des Fonctions Elliptiques et Applications, Chap. 3. 
where $k_{1}, k_{2}=0,1, \cdots, 14$. There are 225 incongruent values of $u$ which include the 9 values of $u$ corresponding to the points of inflexion. The 216 remaining values give the 216 vertices of the 54 quadrilaterals.

The properties of the quadrilaterals may be derived from the matrix given below in which a quadrilateral of a given column corresponds to the point of inflexion represented in the same column. In this table $(i, j)$ denotes $\left(i \omega_{1}+j \omega_{2}\right) / 15$.

\begin{tabular}{r|r|r|r|r|r|r|r|r}
\hline \hline 0,0 & 5,0 & 10,0 & 0,5 & 5,5 & 10,5 & 0,10 & 5,10 & 10,10 \\
\hline 3,0 & 2,0 & 1,0 & 3,5 & 2,5 & 1,5 & 3,10 & 2,10 & 1,10 \\
9,0 & 11,0 & 13,0 & 9,5 & 11,5 & 13,5 & 9,10 & 11,10 & 13,10 \\
12,0 & 8,0 & 4,0 & 12,5 & 8,5 & 4,5 & 12,10 & 8,10 & 4,10 \\
6,0 & 14,0 & 7,0 & 6,5 & 14,5 & 7,5 & 6,10 & 14,10 & 7,10 \\
\hline 3,3 & 2,12 & 1,6 & 3,8 & 2,2 & 1,11 & 3,13 & 2,7 & 1,1 \\
9,9 & 11,6 & 13,3 & 9,14 & 11,11 & 13,8 & 9,4 & 11,1 & 13,13 \\
12,12 & 8,3 & 4,9 & 12,2 & 8,8 & 4,14 & 12,7 & 8,13 & 4,4 \\
6,6 & 14,9 & 7,12 & 6,11 & 14,14 & 7,2 & 6,1 & 14,4 & 7,7 \\
\hline 3,6 & 2,9 & 1,12 & 3,11 & 2,14 & 1,2 & 3,1 & 2,4 & 1,7 \\
9,3 & 11,12 & 13,6 & 9,8 & 11,2 & 13,11 & 9,13 & 11,7 & 13,1 \\
12,9 & 8,6 & 4,3 & 12,14 & 8,11 & 4,8 & 12,4 & 8,1 & 4,13 \\
6,12 & 14,3 & 7,9 & 6,2 & 14,8 & 7,14 & 6,7 & 14,13 & 7,4 \\
\hline 3,9 & 2,6 & 1,3 & 3,14 & 2,11 & 1,8 & 3,4 & 2,1 & 1,13 \\
9,12 & 11,3 & 13,9 & 9,2 & 11,8 & 13,14 & 9,7 & 11,13 & 13,4 \\
12,6 & 8,9 & 4,12 & 12,11 & 8,14 & 4,2 & 12,1 & 8,4 & 4,7 \\
6,3 & 14,12 & 7,6 & 6,8 & 14,2 & 7,11 & 6,13 & 14,7 & 7,1 \\
\hline 3,12 & 2,3 & 1,9 & 3,2 & 2,8 & 1,14 & 3,7 & 2,13 & 1,4 \\
9,6 & 11,9 & 13,12 & 9,11 & 11,14 & 13,2 & 9,1 & 11,4 & 13,7 \\
12,3 & 8,12 & 4,6 & 12,8 & 8,2 & 4,11 & 12,13 & 8,7 & 4,1 \\
6,9 & 14,6 & 7,3 & 6,14 & 14,11 & 7,8 & 6,4 & 14,1 & 7,13 \\
\hline 0,3 & 5,12 & 10,6 & 0,8 & 5,2 & 10,11 & 0,13 & 5,7 & 10,1 \\
0,9 & 5,6 & 10,3 & 0,14 & 5,11 & 10,8 & 0,4 & 5,1 & 10,13 \\
0,12 & 5,3 & 10,9 & 0,2 & 5,8 & 10,14 & 0,7 & 5,13 & 10,4 \\
0,6 & 5,9 & 10,12 & 0,11 & 5,14 & 10,2 & 0,1 & 5,4 & 10,7 \\
\hline \hline
\end{tabular}

The diagonals of any quadrilateral of a given column intersect in the point of inflexion of the same column. Three quadrilaterals of a row which correspond to 3 collinear inflexions are perspective in pairs, the centers of perspectivity being the three collinear inflexions. The point of inflexion at which the diagonals of any one of these quadrilaterals meet is the center of perspectivity of the remaining pair. The 12 vertices of 3 such quadrilaterals are the points of intersection of a quartic with the cubic and lie by threes on 12 straight lines, each line containing a vertex of each quadrilateral. If any 3 of these vertices 
which are collinear are rejected, the remaining 9 are the points of intersection of a cubic with the given cubic. All of these properties may be obtained by employing the relation (1).

The vertices of 3 quadrilaterals in the same column or 3 quadrilaterals corresponding to 3 collinear inflexions, no two of which are in the same row, lie by threes on 4 straight lines.

Two triangles whose vertices are given by $u, u+\omega_{1} / 3$, $u+2 \omega_{1} / 3$, and $-u,-u-\omega_{1} / 3,-u-2 \omega_{1} / 3$, respectively, are seen to be triply in perspective with respect to the 3 collinear inflexions $0, \omega_{1} / 3,2 \omega_{1} / 3$. The vertices of the 2 triangles lie on a conic by relation (1). By assigning appropriate values to $u$ it appears that the 12 vertices of 3 quadrilaterals corresponding to the 3 collinear inflexions $0, \omega_{1} / 3,2 \omega_{1} / 3$ form 2 pairs of triangles, each pair being triply in perspective with respect to these inflexions. The vertices of the triangles of a pair are opposite vertices of the quadrilaterals. Similar relations exist with respect to any 3 collinear inflexions.

If the cubic has a node the elliptic functions reduce to expressions involving trigonometric functions. If we denote the period by $\omega$, the congruence defining $u_{1}$ becomes

$$
15 u_{1} \equiv 0 \bmod \omega,
$$

whence $u_{1}=k \omega / 15$, where $k=0,1, \cdots, 14$. These 15 incongruent values of $u_{1}$ include the parameters of the 3 inflexions. The remaining 12 values give the vertices of 3 quadrilaterals which have the same properties as the first 3 quadrilaterals of the first row in the matrix described above.

If the cubic has a cusp, the elliptic functions reduce to rational functions of $u$, and there are no quadrilaterals since the congruence does not exist.

The University of California 